\title{
NÚCLEO DE ATENÇÃO FARMACÊUTICA: VIVÊNCIAS E PRÁTICAS JUNTO À COMUNIDADE
}

\author{
Daniela Aparecida Martins Trindade \\ Universidade Federal de Alfenas \\ danielaapmtrindade@gmail.com \\ Allen Cristina Ribeiro \\ Universidade Federal de Alfenas \\ allenribeiro332@gmail.com \\ Jefferson Merêncio dos Reis \\ Universidade Federal de Alfenas \\ jef.merencio@hotmail.com
}

Thais Morais Assis Universidade Federal de Alfenas thaismorais_cdm@hotmail.com

Tiago Marques dos Reis Universidade Federal de Alfenas tiagomarques_farmacia@yahoo.com.br

Ricardo Radighieri Rascado Universidade Federal de Alfenas ricardounifal@gmail.com

Luciene Alves Moreira Marques Universidade Federal de Alfenas lualvesmarques@gmail.com

\section{Resumo}

Este artigo apresenta as atividades desenvolvidas e os resultados obtidos pelo Núcleo de Atenção Farmacêutica da Universidade Federal de Alfenas (UNIFAL-MG) nos anos de 2016 a 2017. O Núcleo de Atenção Farmacêutica da UNIFAL é um componente extracurricular para o curso de Farmácia, indispensável à consolidação dos conhecimentos adquiridos durante o curso. Com o farmacêutico atuando nas diversas vertentes da Atenção Farmacêutica, este projeto visa abranger pesquisa, ensino e extensão nesta área, incentivar o trabalho em equipe, promover atividades de ensino curricular e extracurricular, bem como orientar os pacientes quanto ao uso racional dos medicamentos a fim de promover a saúde. O projeto realiza reuniões semanais com discussões de casos clínicos, campanhas educativas para a população e comunidade acadêmica com aferição de parâmetros bioquímicos e fisiológicos, palestras educativas, seminários, eventos científicos como o Simpósio Internacional de Atenção Farmacêutica, Estudos Dirigidos em Atenção Farmacêutica (EDAF), acompanhamento farmacoterapêutico de pacientes portadores de doenças crônicas.

Palavras-chave: Serviços Farmacêuticos. Educação em Saúde. Promoção da Saúde. Estudantes de Farmácia.

\section{PHARMACEUTICAL ATTENTION CENTER: EXPERIENCES AND PRACTICES WITHIN THE COMMUNITY}

Abstract

This article presents the activities developed and the results obtained by the actions of the Nucleus of Pharmaceutical Care of the Federal University of Alfenas (UNIFAL-MG) from 2016 to 2017. The Nucleus of Pharmaceutical Care of UNIFAL is an extracurricular component for the Pharmacy course, indispensable to the consolidation of the knowledge acquired during the course. With the pharmacist acting in the various aspects of Pharmaceutical Care, this project aims to cover research, teaching and extension in this area, encourage teamwork, promote curricular and extracurricular teaching activities, as well as guide patients on the rational use of medicines in order to promote health. The project organizes weekly meetings with discussions of clinical cases, educational campaigns for the population and academic community with biochemical and physiological parameters, educational lectures, seminars, scientific events such as the International Symposium on Pharmaceutical Care, Directed Studies in Pharmaceutical Care (EDAF), pharmacotherapeutic follow-up of patients with chronic diseases.

Keywords: Pharmaceutical Services. Health Education. Health Promotion. Students Pharmacy.

\section{CENTRO DE ATENCIÓN FARMACÉUTICA: EXPERIENCIAS Y PRÁCTICAS EN LA COMUNIDAD}

Resumen

Esto artículo presenta las actividades desarrolladas y los resultados obtenidos por el Núcleo de Atención Farmacéutica de la Universidad Federal de Alfenas (UNIFAL-MG) en los años de 2016-2017. El Núcleo de Atención Farmacéutica de la UNIFAL-MG es un componente extracurricular para el grado de farmacia, indispensable a la consolidación de los conocimientos adquiridos durante el grado. Con el farmacéutico actuando en las diversas vertientes de la Atención Farmacéutica, este proyecto pretende abarcar investigación, enseñanza y extensión en esta área, incentivar el trabajo en equipo, promover actividades de enseñanza curricular y extracurricular, así como orientar a los pacientes en el uso racional de los medicamentos a fin de promover la salud. El proyecto realiza reuniones semanales con discusiones de casos clínicos, campañas educativas para la población y comunidad académica con evaluación de parámetros bioquímicos y fisiológicos, conferencias educativas, seminarios, eventos científicos como el Simposio Internacional de Atención Farmacéutica, Estudios Dirigidos en Atención Farmacéutica (EDAF), seguimiento farmacoterapéutico de pacientes portadores de enfermedades crónicas.

Palabras-clave: Servicios Farmacéuticos. Educación en Salud. Promoción de la Salud. Estudiantes de Farmacia. 
Núcleo de atenção farmacêutica: vivências e práticas junto à comunidade

\section{INTRODUÇÃO}

Nos primórdios da história da Farmácia, os farmacêuticos eram os responsáveis não somente pela produção de medicamentos nas antigas "boticas", mas também pela orientação e acompanhamento dos pacientes no uso desses insumos terapêuticos. Com o surgimento das indústrias farmacêuticas, nas décadas de 1930 e 1940, somado no decorrer dos anos à desvalorização profissional, deficiências de formação e pouca oportunidade de educação continuada, os farmacêuticos assumiram uma posição de meros vendedores de medicamentos, ocasionando perda de identidade profissional (PEREIRA \& FREITAS, 2008; VIEIRA, 2007)

O cuidado farmacêutico foi inicialmente definido por Mikeal e colaboradores em 1975 como sendo o cuidado que um determinado paciente necessita e recebe, que promove o uso seguro e racional dos medicamentos (CIPOLLE, 2006). Brodie et al. (1980) e Hepler (1988) ampliaram a definição elaborada anteriormente; porém, foram Hepler \& Strand que pela primeira vez introduziram o conceito de "pharmaceutical care". De acordo com Hepler \& Strand (1990, apud CIPOLLE, 2006): “o cuidado farmacêutico é um componente do exercício profissional do farmacêutico que impõe uma interação direta do farmacêutico com o paciente, com o propósito de cuidar das necessidades deste em relação aos medicamentos".

O termo "atenção farmacêutica" (pharmaceutical care) foi introduzido no Brasil para designar diferentes serviços clínicos, mas também para referir-se a um único serviço - o acompanhamento farmacoterapêutico (CFF, 2016). Segundo Zubioli (2007, apud CFF, 2016), como os espanhóis traduziram o termo pharmaceutical care por atención farmacéutica, o termo "atenção farmacêutica" foi introduzido no Brasil como uma tradução literal do termo espanhol, porém, sem o mesmo significado do termo "pharmaceutical care". A Ordem dos Farmacêuticos e a Associação Nacional das Farmácias de Portugal utilizam o termo "cuidado farmacêutico", uma vez que na origem latina "atenção" e "cuidado" têm significados diferentes.

Desde 1995, o termo “atenção farmacêutica, o qual foi equivocadamente traduzido do termo "pharmaceutical care", vem sendo discutido e abordado por instituições de educação, saúde e de representação profissional (CRF-SP, 2010). Recentemente, o tema tem sido amplamente discutido pelo Conselho Federal de Farmácia que regulamentou as atividades clínicas do farmacêutico por meio de resoluções (CFF, 2013a; CFF, 2013b).

Todas estas mudanças geraram uma alteração conceitual, estrutural e filosófica nos cursos de Farmácia que passaram a ter uma formação generalista, podendo o farmacêutico atuar em vários segmentos e atividades de acordo com sua formação profissional. Atualmente existem 131 especialidades da profissão distribuídas em 10 áreas de atuação regulamentadas pela Resolução 
Núcleo de atenção farmacêutica: vivências e práticas junto à comunidade

CFF $n^{\circ} 572 / 2013$, enfatizando os temas relacionados a aspectos sanitários e sociais, incluindo a atenção farmacêutica (PEREIRA \& FREITAS, 2008; CFF, 2013c ; OLIVEIRA et al., 2005). Recentemente, foi publicada uma nova Diretriz Curricular Nacional (BRASIL, 2017) para o curso de Farmácia, dada a necessária articulação entre conhecimentos, competências, habilidades e atitudes, para contemplar o perfil do egresso. Para alcançar tal objetivo, a formação foi estruturada em três eixos: Cuidado em Saúde, Tecnologia e Inovação em Saúde, e Gestão em Saúde. Entende-se, como cuidado em saúde, o conjunto de ações e de serviços ofertados ao indivíduo, à família e à comunidade, que considera a autonomia do ser humano, a sua singularidade e o contexto real em que vive, sendo realizado por meio de atividades de promoção, proteção e recuperação da saúde, além da prevenção de doenças, e que possibilite às pessoas viverem melhor (BRASIL, 2017).

Uma formação mais humanística e ao mesmo tempo menos tecnicista torna-se fundamental para o farmacêutico, pois busca atender uma nova demanda social, sendo a atenção farmacêutica ou o cuidado farmacêutico, prática essencial nos serviços de saúde (PEREIRA \& NASCIMENTO, 2011). Esta formação pode ser concretizada a partir de uma prática associada a um suporte pedagógico voltado às necessidades da população, uma vez que as situaçõesproblema vivenciadas no dia a dia exigem ações que vão além do caráter puramente teóricocientífico (COSTA et al., 2014).

De acordo com o Conselho Federal de Farmácia, o cuidado centrado no paciente proposto no cuidado farmacêutico é a relação humanizada que envolve o respeito às crenças, expectativas, experiências, atitudes e preocupações do paciente ou cuidadores quanto às suas condições de saúde e ao uso de medicamentos, na qual farmacêutico e paciente compartilham a tomada de decisão e a responsabilidade pelos resultados em saúde alcançados (CFF, 2016). O farmacêutico, embora seja um profissional importante da área da saúde vem sendo subutilizado nos sistemas públicos e até mesmo no sistema privado. Prover cuidado prevenindo os problemas relacionados aos medicamentos e resultados clínicos negativos associados ao uso desses em relação ao paciente, às famílias e à comunidade torna-se um desafio (CFF, 2016). Segundo dados do DataSus (2013, apud CFF 2016), podem ter ocorrido entre 1,2 milhão e 3,2 milhões de internações de urgência ligadas a problemas relacionados aos medicamentos, resultando em um custo estimado de $\mathrm{R} \$ 1,3$ bilhões e R $\$$ 3,6 bilhões. Estudos realizados em 2002 demonstraram que $28 \%$ das visitas ao pronto socorro, estavam relacionados a problemas na farmacoterapia, e destes, $70 \%$ eram evitáveis.

De acordo com Gastelurrutia et al. (2005), serviços farmacêuticos são serviços destinados ao paciente e realizados por farmacêuticos que, exigindo conhecimentos específicos, têm por 
Núcleo de atenção farmacêutica: vivências e práticas junto à comunidade

objetivo melhorar o processo de uso dos medicamentos. Também pode ser definido como: "Um conjunto de atividades organizadas em um processo de trabalho, que visa a contribuir para prevenção de doenças, promoção, a proteção e recuperação da saúde, e para a melhoria da qualidade de vida das pessoas" (CFF, 2016). O Conselho Federal de Farmácia, após extensa revisão da literatura, apresenta em sua publicação "Programa de Suporte ao Cuidado Farmacêutico na Atenção à Saúde" (PROFAR) um elenco de nove serviços farmacêuticos que podem ser oferecidos: rastreamento em saúde, educação em saúde, manejo de problemas de saúde autolimitados, dispensação, monitorização terapêutica de medicamentos, conciliação de medicamentos, revisão da farmacoterapia, gestão da condição de saúde e acompanhamento farmacoterapêutico (CFF, 2016).

Diante do exposto, o Núcleo de Atenção Farmacêutica (NAFAU) da Universidade Federal de Alfenas (UNIFAL-MG) tem por objetivo realizar e preparar estudantes do curso de Farmácia para os serviços farmacêuticos. O NAFAU é um Programa de Extensão Universitária composto por 16 membros, discentes do curso de Farmácia, sendo 15 mulheres com idade entre 21 - 26 anos. Foi fundado no ano de 2012 por um acadêmico de Farmácia e desde a fundação até o presente teve 49 membros (contando com os atuais). O grupo está sob a coordenação de dois professores e conta com a colaboração de um terceiro professor, todos da UNIFAL-MG. Semanalmente, os membros realizam reuniões com duração média de uma hora, para delinear as atividades que serão desenvolvidas ao longo do semestre. Os membros do grupo são selecionados por avaliação escrita, análise de curriculum e entrevista (com dinâmicas de grupo) e sua permanência no grupo se dá por dois anos podendo ser estendida a critério do grupo. $\mathrm{O}$ acadêmico que tiver três faltas não justificadas (entenda-se por justificativa: atestado médico e provas no horário das atividades) em atividades do grupo será excluído do mesmo.

Atualmente, o NAFAU oferece em suas atividades três serviços farmacêuticos: a) educação em saúde que consiste em ações que visam integrar o conhecimento científico e popular no intuito de dar autonomia aos pacientes em relação aos problemas de saúde e seu tratamento; b) rastreamento em saúde que consiste de ações que possibilitam a identificação de doença precoce em pessoas assintomáticas ou que possuem algum risco de desenvolvê-la, e; c) acompanhamento farmacoterapêutico que consiste no gerenciamento da farmacoterapia com o objetivo de torná-la efetiva e segura, alcançando assim melhores resultados clínicos.

Este artigo tem por objetivo fazer um relato das experiências do NAFAU em relação aos serviços farmacêuticos prestados ao paciente, à família e à comunidade nos anos de 2016 e 2017. 


\section{MATERIAIS E MÉTODOS}

Este artigo se trata de um estudo descritivo qualitativo na modalidade relato de experiência.

A população abrangida nesse estudo foi a comunidade interna à UNIFAL-MG, formada por acadêmicos de diversos cursos de graduação e pós-graduação, funcionários públicos e terceirizados e pela comunidade externa à referida instituição de ensino (caracterizada pela população de Alfenas-MG).

O NAFAU desenvolve ações de ensino, pesquisa e extensão. Entre as atividades de extensão estão palestras e minicursos no campus universitário com temas pertinentes e atualidades da área de farmácia clínica para complementação da grade curricular dos graduandos, campanhas de orientação em saúde e o programa "Atenfar no seu lar".

Durante as campanhas de orientação em saúde foram realizadas aferições de parâmetros bioquímicos, fisiológicos e antropométricos, entre eles: glicemia capilar, triglicérides e colesterol total capilar, aferição de pressão arterial, determinação do índice de massa corporal, além de orientar quanto ao uso racional de medicamentos.

O "AtenFar no seu lar" é um programa que objetiva o acompanhamento farmacoterapêutico do paciente e vem sendo desenvolvido no domicílio dos pacientes. Os pacientes são recrutados através das campanhas de saúde realizadas pelo Núcleo. Aqueles pacientes que apresentam algum parâmetro bioquímico, fisiológico ou antropométrico alterado ou que demonstram possuir alguma dúvida em relação ao seu tratamento são convidados para participar do acompanhamento. Caso aceite, cada paciente é acompanhado por uma dupla de acadêmicos que utiliza como ferramenta o Método Dáder no acompanhamento farmacoterapêutico. Este método consiste em uma primeira visita, na qual o farmacêutico coleta dados sobre a história farmacoterapêutica do paciente. Em seguida, com os dados coletados, elabora-se o estado de situação do paciente (resumo do caso), identificam-se problemas relacionados aos medicamentos que podem gerar resultados clínicos negativos e; elabora-se um plano de intervenção para o paciente. Em uma segunda visita, o paciente é informado do plano de cuidado e este é colocado em prática. A terceira visita é realizada no intuito de verificar se o problema foi resolvido ou se haverá necessidade de aguardar um tempo maior, sendo então realizada uma quarta visita. Os pacientes receberam uma visita mensal, totalizando quatro visitas por semestre. 
Núcleo de atenção farmacêutica: vivências e práticas junto à comunidade

As atividades de extensão desenvolvidas pelo NAFAU estão aprovadas pelo Comitê de Ética em pesquisa com seres humanos da Universidade Federal de Alfenas, sob o parecer n. 114.294.

As experiências vivenciadas pelo NAFAU aqui relatadas se referem ao período de fevereiro de 2016 a setembro de 2017.

\section{RESULTADOS E ANÁLISES}

No final de 2016 foram realizados pelos acadêmicos participantes do grupo do NAFAU os Estudos dirigidos em Atenção Farmacêutica (EDAF), com os seguintes temas: atenção farmacêutica ao paciente com HIV, atenção farmacêutica ao paciente com síndrome metabólica, atenção farmacêutica para a gestante, atenção farmacêutica à saúde do homem e da mulher. Durante a realização do evento foi atingido um público de 200 participantes nos quatro dias de palestras realizadas na UNIFAL-MG. Os públicos-alvo foram acadêmicos e profissionais de farmácia.

Em 2016, o NAFAU participou da Campanha Farmacêuticos em ação: todos contra o Aedes aegypti, organizada pelo Conselho Federal de Farmácia. A campanha foi realizada em abril e setembro na praça da cidade, na qual foram fornecidas orientações sobre os cuidados que as pessoas devem ter para evitar a proliferação dos mosquitos Aedes aegypti e Aedes albopictus, e assim evitar que as doenças Dengue, Chikungunya e Zika continuassem sendo propagadas. Foram entrevistadas 89 pessoas, destas 69,66\% do gênero masculino, com idade média de 57 anos $\pm 14,63$. Destas 89 pessoas, $22 \%$ afirmaram saber diferenciar os sintomas das doenças Dengue, Chikungunya e Zika. Doze por cento afirmaram já ter tido dengue, 94\% dos entrevistados fazem o combate contra os mosquitos (evitando acúmulo de água parada em casa), 25\% fazem uso de repelente e $70 \%$ usam inseticida em casa.

No final do semestre de 2015 e início de 2016, o NAFAU realizou uma campanha dentro do campus e na praça da cidade com a distribuição de cerca de 1500 folders educativos sobre o tema: Fotoeducação: orientações farmacêuticas no combate ao câncer de pele. As pessoas foram solicitadas a responder uma enquete sobre fotoproteção. Duzentas e trinta e cinco pessoas responderam à enquete, porém, apenas 40,85\% dos respondentes relataram fazer uso regular de protetor solar. Entre os que não faziam o uso diário de protetor solar, um dos principais motivos para o não uso era o esquecimento (74 participantes), mesmo tendo o produto em suas residências; diferentemente do que a maioria esperava, os participantes sabiam da importância mas mesmo assim não faziam o uso de protetor solar diariamente. Além da campanha, o 
Núcleo de atenção farmacêutica: vivências e práticas junto à comunidade

NAFAU ministrou palestra sobre o uso de fotoprotetores para a população de Alfenas atendida pelo CRAS- Centro de Referência e Assistência Social do bairro Campos Elíseos (gestantes, adolescentes, crianças e idosos).

A campanha "Setembro Amarelo" contou com a participação de vários acadêmicos (aproximadamente 50 acadêmicos de diversos cursos de graduação: Farmácia, Enfermagem, Letras, Odontologia, Geografia). As atividades desenvolvidas foram oficina de desenho, apresentação musical do grupo Batuki mental composto por pacientes do Centro de Atenção Psicossocial de Alfenas, dança circular, alongamento e massagem e animação com os doutores da alegria (acadêmicos do projeto Cuidando da gente) (Figura 1).

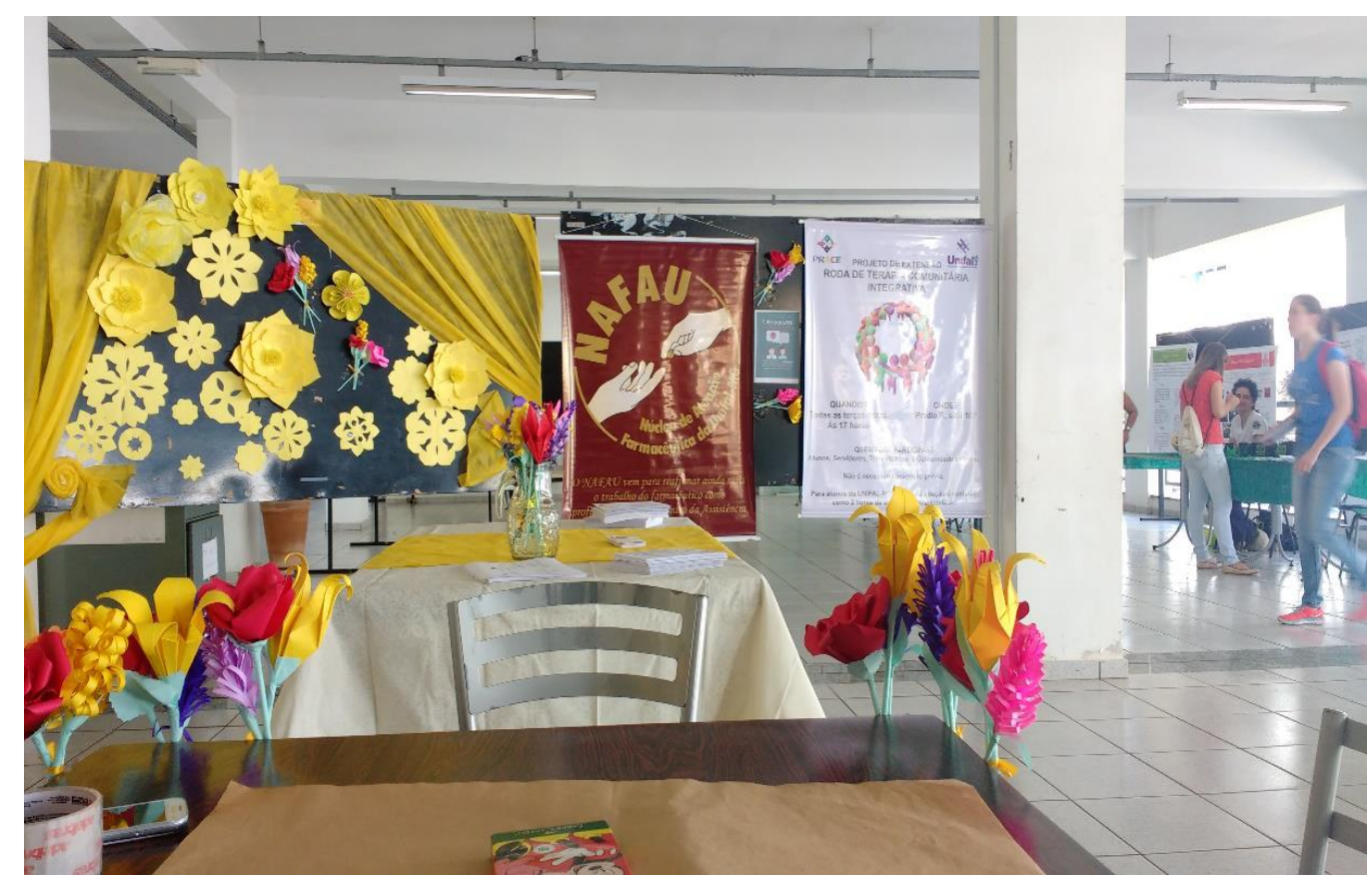

Figura 1: Campanha "Setembro Amarelo" no campus universitário.

Outro serviço farmacêutico prestado pelo NAFAU consiste no rastreamento em saúde. Este rastreamento tem por objetivo identificar pacientes que não tenham ainda um diagnóstico. Cabe salientar que todos os pacientes que apresentam um parâmetro alterado são encaminhados ao médico para que sejam realizados outros exames e elaborado o diagnóstico. Embora o rastreamento em saúde possa ser realizado por qualquer profissional da saúde, torna-se importante que o farmacêutico no processo de cuidado realize tal serviço farmacêutico (CFF, 2016). O serviço de rastreamento oferecido pelo Núcleo abrange não apenas a comunidade interna da UNIFAL, mas também a comunidade externa através de campanhas realizadas durante todo o ano. 
Durante a campanha pelo uso racional de medicamentos realizada este ano no centro de convivência da cidade de Alfenas-MG, foram realizados 250 testes de glicemia capilar e 250 aferições de pressão arterial como rastreamento em saúde. Aqueles pacientes que apresentaram alguma alteração $(10 \%)$ foram encaminhados ao médico para diagnóstico (quando relatavam não utilizar nenhum tipo de medicamento) ou ao consultório farmacêutico para acompanhamento farmacoterapêutico (quando relatavam utilizar medicamentos para hipertensão e/ou diabetes).

Até o momento, foram atendidos sete pacientes pelo projeto "Atenfar no seu lar", projeto no qual são realizadas visitas domiciliares com o objetivo de realizar o acompanhamento farmacoterapêutico de pacientes. Destes sete pacientes, as doenças mais citadas foram a hipertensão seguida do hipotireoidismo. De acordo com o relato dos próprios pacientes, todos aderem $100 \%$ ao tratamento e todos apresentam conhecimento sobre sua doença. Foram realizadas cinco intervenções farmacêuticas, sendo os cinco problemas encontrados resolvidos após intervenção. Os problemas encontrados estavam relacionados a interações medicamentoalimento $(n=3)$ e reações adversas $(n=-2)$, classificados respectivamente como resultados clínicos negativos (RNM) do tipo inefetividade quantitativa e insegurança não quantitativa. Há a intenção, para o ano de 2018, de que o serviço de acompanhamento farmacoterapêutico seja oferecido em uma Farmácia do SUS para pacientes portadores de doenças crônicas, pois o fato de o serviço ser oferecido no domicílio acarreta algumas dificuldades para os acadêmicos, pois estes possuem uma carga horária elevada e precisar se deslocar até o domićlio dos pacientes consome o dobro do tempo em relação a se o paciente estivesse sendo atendido em uma farmácia. Outra dificuldade se refere aos custos, pois os acadêmicos não têm nenhum incentivo para custear seu deslocamento.

Considerando a possibilidade de maior abrangência através do ambiente virtual e o crescente uso de ferramentas online como fonte de conhecimento e informação, o Núcleo criou um blog e um perfil na rede social Facebook, com atualização semanal, abordando notícias e atualidades na área da saúde, baseando-se em evidências científicas a fim de sanar dúvidas e difundir conhecimentos com assuntos pertinentes da área farmacêutica e clínica, sendo estas publicações supervisionadas e revisadas pela coordenadora do NAFAU. Nos anos de 2016 e 2017, o blog teve 9.898 e 2816 acessos respectivamente; e, o perfil no Facebook possui 1354 seguidores. Os últimos temas abordados pelo Blog foram: glaucoma, saúde ocular, estigma em saúde mental, hipertensão arterial, queimaduras, acidentes de trabalho, semana mundial da amamentação, semana de cessação do tabagismo, orientações para o autoteste para HIV disponível nas farmácias e drogarias, Setembro Amarelo (campanha de prevenção ao suicídio). 
Núcleo de atenção farmacêutica: vivências e práticas junto à comunidade

As atividades desenvolvidas pelo NAFAU estão em consonância ao que preconiza a Resolução 585/2013 do Conselho Federal de Farmácia, que define as atribuições clínicas do farmacêutico (CFF, 2013a).

\section{CONSIDERAÇÕES FINAIS}

Impossível pensar em saúde sem pensar em educação e nas relações que existem entre elas (GAZZINELLI et al., 2006). É nesse contexto que se insere a importância do farmacêutico como educador em saúde, capaz de atuar em defesa da saúde do usuário através da promoção do uso racional de medicamentos, proteção e recuperação da saúde do paciente, da família e da comunidade.

A educação em saúde envolve o empoderamento do paciente e traz como eixo fundamental a troca de experiências e saberes entre profissional e paciente ou comunidade (CFF, 2016a). O NAFAU tem contribuído nesse sentido, pois tanto em suas campanhas de educação em saúde quanto durante o processo de cuidado farmacêutico (Atenfar no seu lar), este tem buscado empoderar o indivíduo e educá-lo de acordo com seu nível de conhecimento, crenças e experiências prévias, não se constituindo, pois, em um ato impositivo.

É bastante evidente o quão importante é o trabalho do farmacêutico com a comunidade envolvendo-se não só na dispensação dos medicamentos, mas atuando também de forma educativa (MEROLA et al., 2005).

A falta de orientação é vista como um obstáculo para a prevenção de doenças e suas complicações, e acarreta um alto custo para a saúde pública (CRFSP, 2017). Considerando que hoje é cada vez mais crescente uso de ferramentas virtuais como fonte de ensino e informação, o Núcleo elabora boletins quinzenais e divulga informação relevante na área da saúde.

Segundo Vermelho et al. (2014), a cada dia aumenta a crença de que as tecnologias de comunicação em rede podem ser uma ferramenta eficiente para promover a comunicação, pois se constituem na ferramenta mais poderosa de circulação de informação da contemporaneidade. Ainda, foi observado que a área da saúde é uma das que contém mais informações disponíveis e acessadas por uma quantidade cada vez maior de pessoas: oito em cada dez internautas já acessaram sites de informações de saúde; no Brasil, estima-se que cerca de dez milhões de internautas acessam regularmente sites de informações de saúde (LIMA et al., 2015). Isso reforça a importância do Blog e do perfil no Facebook. O Blog do NAFAU com aproximadamente 13.000 acessos se considerarmos 2016 e 2017 tem conseguido difundir informação de qualidade na área da saúde e do cuidado farmacêutico. 
Núcleo de atenção farmacêutica: vivências e práticas junto à comunidade

As ações de extensão desenvolvidas pelo NAFAU aproximam o acadêmico da realidade que o cerca, contribuem para o desenvolvimento de uma interação ensino-serviço e para o desenvolvimento de habilidades de comunicação nos discentes.

O NAFAU permite estabelecer uma relação dialógica entre a Universidade e comunidade, através da troca de experiências e saberes. Esta relação permite ao acadêmico aproximar-se do contexto social e aplicar os conhecimentos teóricos adquiridos, para melhor desenvolver-se como profissional.

Os desafios encontrados durante a execução das atividades referem-se principalmente à falta de recursos financeiros para execução das mesmas. Outro desafio a ser superado é conciliar as atividades do programa com a carga horária elevada dos acadêmicos do curso de Farmácia. No entanto, apesar disso, temos conseguido executar as atividades com recursos próprios (muitas vezes) e a maioria das atividades é realizada à noite ou aos sábados e até mesmo aos domingos. As próximas atividades propostas para o NAFAU incluem duas campanhas de grande importância nacional: Outubro Rosa e Novembro Azul, a implantação do acompanhamento farmacoterapêutico em uma farmácia do SUS e o engajamento dos acadêmicos na área da saúde mental, desenvolvendo projetos de extensão e pesquisa nessa área.

O Programa contribuiu para a multiplicação do conhecimento em saúde tanto para a equipe integrante quanto para a comunidade interna e externa à UNIFAL-MG, utilizando também as redes sociais como ferramenta de informação. Os serviços oferecidos ao paciente, à família e à comunidade são ações desenvolvidas continuamente pelo NAFAU, não apenas nos anos de 2016 -2017, mas desde sua fundação em 2012.

Financiamento: PROBEXT-UNIFAL 2017

Agradecimentos: À Pró-Reitoria de Extensão da Universidade Federal de Alfenas, e aos demais membros do NAFAU: Aline Karina Maure, Bruno Ewerton Meireles Souto, Camila Campos Dutra, Giuliana Martina Castorani, Josiane Aparecida Rocha, Laira Maria Faria Matias, Luana Silva Ferreira, Michelle de Jesus Coimbra. 


\section{REFERÊNCIAS}

BRASIL, Ministério da Educação, Conselho Nacional de Educação, \& Câmara de Educação Superior. (2017). Resolução $\mathrm{n}^{\circ} 6$ de 19 de outubro de 2017. Institui diretrizes curriculares nacionais do curso de graduação em Farmácia e dá outras providências. Diário Oficial da União.

BRODIE, D.C.; PARISH, P.A.; POSTON, J.W. Societal needs for drugs and drug-related services. Am. J. Pharm. Educ., v.44, n.3, p.276-78, 1980.

CFF. Conselho Federal de Farmácia. Resolução 585 de 23 de agosto de 2013. Regulamenta as atribuições clínicas do farmacêutico e dá outras providências. Brasília, 2013a. Disponível em: http://www.cff.org.br/userfiles/file/resolucoes/585.pdf. Acesso em: 20 set. 2016.

CFF. Conselho Federal de Farmácia. Resolução 586 de 29 de agosto de 2013. Regula a prescrição farmacêutica e dá outras providências. Brasília, 2013b. Disponível em: http://www.cff.org.br/userfiles/file/resolucoes/586.pdf. Acesso em: 20 set. 2017.

CFF. Conselho Federal de Farmácia. Resolução no 572 DE 25 DE ABRIL DE 2013 Dispõe sobre a regulamentação das especialidades farmacêuticas, por linhas de atuação. Brasília, 2013c. Disponível em: http://www.cff.org.br/userfiles/file/resolucoes/572.pdf. Acesso em: 22 jul. 2018.

CFF. Serviços farmacêuticos diretamente destinados ao paciente, à família e à comunidade: contextualização e arcabouço conceitual / Conselho Federal de Farmácia. - Brasília: Conselho Federal de Farmácia, 2016. 200 p.

CIPOLLE, R.J. O exercício do cuidado farmacêutico. Robert J. Cipolle, Linda M. Strand, Peter C. Morley. Tradução: Denise Borges Bittar; Revisao técnica: Arnaldo Zubioli- Brasília: Conselho Federal de Farmácia, 2006. 396 p.

COSTA, E.M.; ROCHA, A.; RABELO, D.M.; LIMA, J.G. Avaliação do papel do farmacêutico nas ações de promoção da saúde e prevenção de agravos na atenção primária. Revista Ciências Farmacêuticas Básica Aplicada, v. 35, n. 1, p. 81-8, 2014.

CRFSP. Conselho Regional de Farmácia do Estado de São Paulo. Campanhas de educação em saúde. Disponível em: <http://www.crfsp.org.br/88-nep/campanhas/863-campanhas-deeducacao-em-saude.html>. Acesso: 30 de jul. 2017.

CRFSP. Conselho Regional de Farmácia do Estado de São Paulo. Organização Pan-Americana da Saúde Fascículo III - Serviços Farmacêuticos / Projeto Farmácia Estabelecimento de Saúde / CRF-SP: Conselho Regional de Farmácia do Estado de São Paulo; Organização Pan-Americana de Saúde - Brasília, 2010.

GASTELURRUTIA MA, FERNÁNDEZ-LLIMÓS F, GARCÍA DELGADO P, GASTELURRUTIA P, FAUS MJ, BENRIMOJ SI. Barriers and facilitators to the dissemination and implementation of cognitive services in Spanish community pharmacies. Seguimiento Farmacoterapeutico, v.3, n.2, p. 65-77, 2005.

GAZZINELLI, M. F; REIS, D. C.; MARQUES, R. C. Educação em saúde: teoria, método e imaginação. Belo Horizonte: Ed UFMG, 2006. 166 p. 
Núcleo de atenção farmacêutica: vivências e práticas junto à comunidade

HEPLER, C.D. The third wave in pharmaceutical education: the clinical movement . Am. J. Pharm. Educ., v.51, n.4, p.369-385, 1987.

HEPLER, C.D.; STRAND, L.M. Opportunities and responsibilities in pharmaceutical care. Am. J. Hosp. Pharm., v.47, n.3, p.533-543, 1990.

LIMA, S.G.; GUIMARÃES, K.M.; GERHARDT, P.C.; CASSIANO, A.C.; VERMELHO, S.C.; BORTOLOZZI, F. A Utilização de Redes Sociais Digitais na Área da Saúde: Uma Revisão Sistemática. Saúde e Pesquisa, v. 30, n.8, p. 93-101, 2015.

MEROLA, Y.D.L; EL-KHATIBS.; GRANJEIRO, P.A. Atenção Farmacêutica como Instrumento de Ensino. Infarma, v.17, n.7/9, p. 70-72, 2005.

OLIVEIRA, A.B. et al. Obstáculos da atenção farmacêutica no Brasil. Revista Brasileira de Ciências Farmacêuticas. v. 41, n. 4, p. 409-413, 2005.

PEREIRA, L.R.L; FREITAS, O. A evolução da Atenção Farmacêutica e a perspectiva para o Brasil. Revista Brasileira de Ciências Farmacêuticas. v. 44, n. 4, p. 601-612, 2008.

PEREIRA, M.L.; NASCIMENTO, M.M.G. Das boticas aos cuidados farmacêuticos: perspectivas do profissional farmacêutico. Rev. Bras. Farm, v. 92, n. 4, p. 245-252, 2011.

VERMELHO, S.C.; VELHO, A.P.M.; BONKOVSK, A.; PIROLA, A. Refletindo sobre as redes sociais. Educação \& Sociedade, v. 35, n. 126, p. 179-196, 2014.

VIEIRA, S.V. Possibilidades de contribuição do farmacêutico para a promoção da saúde. Ciência \& Saúde Coletiva, v. 12, n. 1, p. 213-220, 2007.

Recebido em: 22/01/2019

Aceito em: 17/05/2019 